\title{
LA INVESTIGACIóN EN LA ZONA SUR: DE LA MANO CON EL DESARROLLO DE LA REGIóN
}

\author{
Milena Alcócer Tocora ${ }^{65}$
}

RESUMEN

La UNAD, dentro de su horizonte institucional, ha definido el desarrollo regional como uno de sus ejes fundamentales. En este sentido, los acercamientos que se tengan con los diferentes estamentos de la sociedad en cada una de las regiones son relevantes para dar cumplimiento y ser consecuente con el quehacer social que como Universidad debe propender.

El presente artículo tiene como objetivo dar a conocer la dinámica y los resultados de investigación que se han generado en la zona sur; se identifica geográfica, cultural y socialmente la región junto con cada uno de los centros que la componen, se socializa la dinámica del Sistema de Investigación Unadista (SIUNAD) y los principales productos obtenidos del trabajo de redes, grupos y semilleros de investigación. En este sentido se revela la prospectiva que permitirá a esta zona posicionarse a nivel nacional como un nodo potencializador de conocimiento científico que dará respuestas contundentes a las problemáticas de la sociedad Colombiana.

Palabras clave: desarrollo regional, investigación, redes.

\begin{abstract}
The UNAD within its institutional horizon has defined the regional development as one of its fundamental axes. In this sense, the approaches being taken to different levels of society in each of the regions are relevant to comply and be consistent with the social activity which a University must strive.

This article aims to present the dynamics and results of research that have been generated in the South Zone; identifying geographical, cultural and social the region with each component that institute it, dynamics of the Unadista Research System (SIUNAD) is socialized and the main products obtained from the work of networks, groups and seeds researchers, in this sense it is revealed the foresight to allow the South Zone position nationally as a node propelling of scientific knowledge that will give conclusive answers to the problems of Colombian society.
\end{abstract}

Keywords: Regional Development, Research, Networks.

Recibido: 28 de abril de 2010 Aceptado: 24 de mayo de 2010

65 Especialista en química de productos naturales, Coordinadora Zonal Investigación-Zona Sur. Nodo CEAD Ibagué. Correo: milena.alcocer@unad.edu.co 


\section{IDENTIfICACIóN y CARACTERIZACIóN DE LA ZONA SUR}

La nueva Zona Sur se consolida en el año 2009 y se encuentra integrada por los Departamentos de Tolima, Huila y Caquetá:

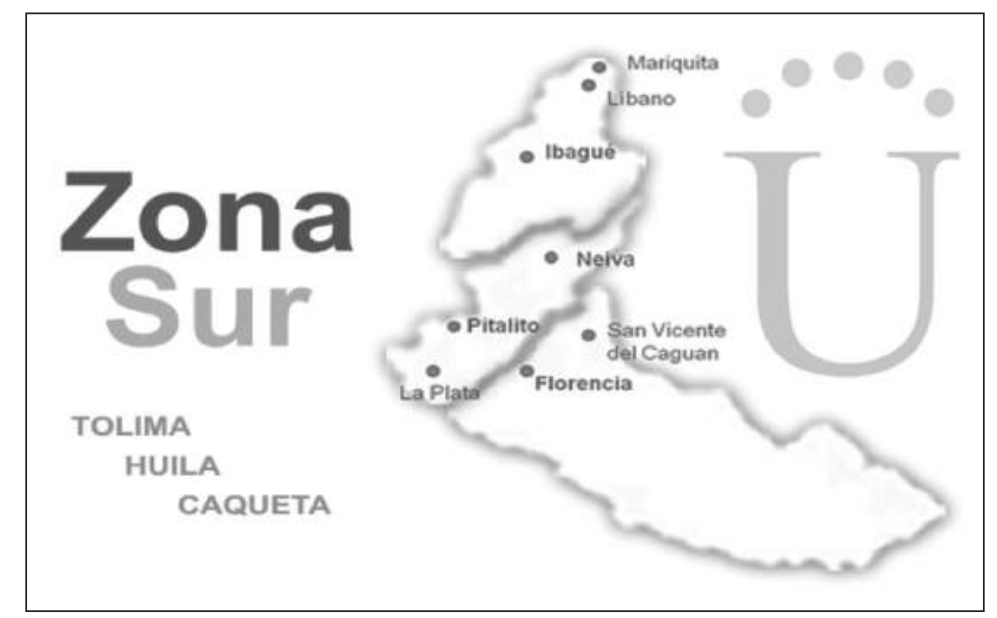

figura 1. Zona Sur

\section{Tolima:}

Se sitúa en la región Andina, centro-occidente del país; limita por el norte y el oriente con el Departamento de Caldas; por el oriente con el departamento de Cundinamarca; por el sur con los Departamentos del Huila y Cauca, y por el occidente con los departamentos de Valle del Cauca, Quindío y Risaralda; tiene una superficie de $23.562 \mathrm{~km}^{2}$ y su capital es Ibagué, conocida como la Ciudad Musical de Colombia.

El CEAD de Ibagué: en 1987 la UNAD llega al Departamento del Tolima en un momento crucial para el desarrollo del talento humano de la región y en 1990 consigue su propia sede; desde el año 2009, el CEAD se convierte en el Nodo de la Zona Sur, que actualmente, atiende alrededor de 1.416 estudiantes de pregrado.

La investigación en el CEAD ha venido consolidándose a través de la dinamización de proyectos desde cada una de las escuelas. En este sentido, sus investigadores han participado en las convocatorias internas de financiación de proyectos, organizadas por el SIUNAD; es así como el Grupo de Investigación en Etnofarmacología productos naturales y alimentos GIEPRONAL, se destaca por la generación de proyectos importantes para el sector agropecuario y de la salud, al igual que por su participación en la Semana Departamental de Ciencia y Tecnología Organizada por el CODECyT. 


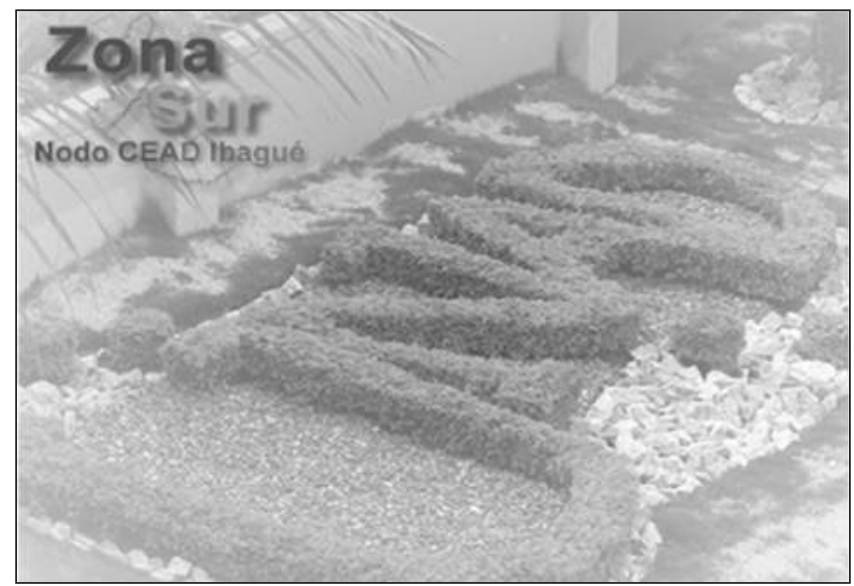

figura 2. CEAD Ibagué

La UNAD en el Tolima hace presencia en los CERES

\section{Líbano:}

El municipio del Líbano, se localiza al norte del Departamento del Tolima, posee una extensión de $299.44 \mathrm{Km} 2$, su temperatura media es de $20^{\circ} \mathrm{C}$ y se encuentra ubicado a 120 Kilómetros de la Ciudad de Ibagué. Se configuró como aldea hacia 1.886; a partir de este momento y hasta 1900, el Líbano se desarrolló luchando contra la selva, abriendo caminos de herradura por todas las direcciones, ubicándose al final de este periodo en la categoría de provincia, que incluía los actuales municipios de Villa Hermosa, Casabianca, Fresno, Herveo y Santa Isabel, con aproximadamente 2.000 habitantes.

El desarrollo socio económico de la región, fue orientado por sus fundadores hacia la explotación de un importante potencial minero y al cultivo del café, el cual ha llegado a ser el elemento característico y principal de la economía, sociedad y cultura libanense.

El CERES registra actualmente un número de 187 estudiantes. Su actividad investigativa es naciente, en el 2009 inicia su integración al Sistema de Investigación Unadista, a través de la generación de dos semilleros de investigación conformados por estudiantes de las Escuelas de Ciencias Sociales Artes y Humanidades (ECSAH) y de Ciencias Agrícolas Pecuarias y del Medio Ambiente (ECAPMA). 


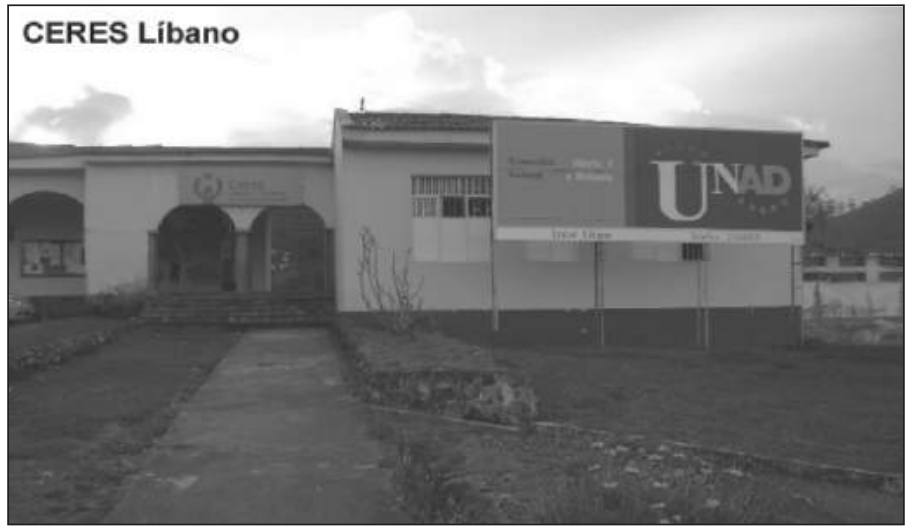

figura 3. CERES Líbano

\section{San Sebastián de Mariquita:}

El municipio de San Sebastián de Mariquita limita con los Municipios de Honda, Armero Guayabal y Fresno; tiene una extensión de $37.952 \mathrm{Km} 2$ y una temperatura promedio de de $27^{\circ} \mathrm{C}$.

Sirvió de sede de la llamada Real Expedición Botánica, ordenada por el rey Carlos III, bajo la dirección de José Celestino Mutis, quien dirigió el estudio de la flora del lugar en unión con científicos criollos como Francisco José de Caldas.

El CERES a la fecha atiende alrededor de 298 estudiantes. Durante el año 2009 logró consolidar un semillero de investigación integrado a la Escuela de Ciencias Sociales Artes y Humanidades (ECSAH); este semillero ha representado a la universidad en el encuentro departamental de semilleros 2009.

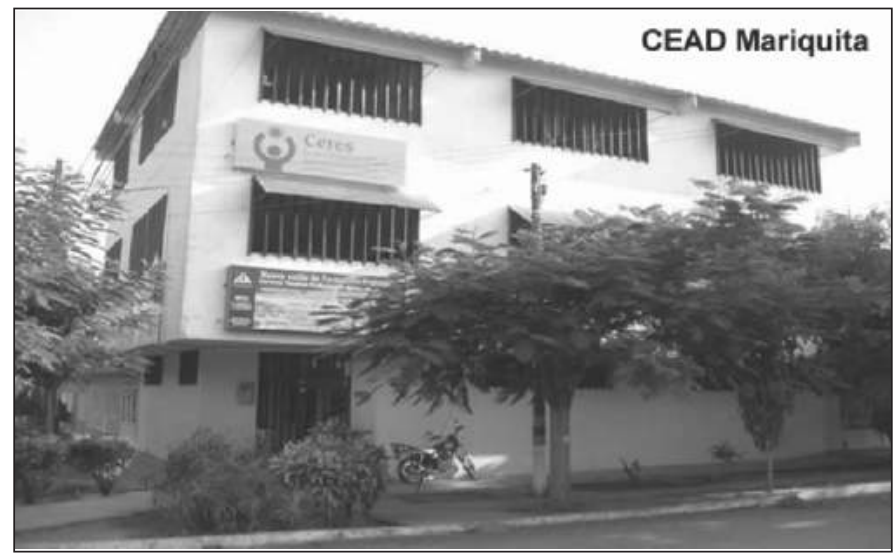

figura 4. CERES San Sebastián de Mariquita 


\section{Huila}

Perteneció al denominado Tolima Grande o Alto Magdalena, territorio formado por los Departamentos de Tolima y Huila. Su historia está sustentada en el legado de la cultura Agustiniana, cuya importancia radica en la categoría de Patrimonio Cultural de la Humanidad (entre otros aspectos, por los parques Arqueológicos de San Agustín, Alto de Los Ídolos y Alto de Las Piedras) reconocida por la UNESCo en 1995.

En el Departamento del Huila la UNAD hace presencia en los siguientes municipios a través de lOS CCAV, CEAD Y CERES.

\section{CCAV Neiva:}

Neiva es la capital del Departamento del Huila, se ubica entre la Cordillera Central y Oriental, en una planicie sobre la margen oriental del río Magdalena; su extensión territorial es de 1.533 $\mathrm{Km}^{2}$, su altura de 442 metros sobre el nivel del mar y su temperatura promedio es de $27^{\circ} \mathrm{C}$.

La ciudad cuenta con una población aproximada de 315.332 habitantes, correspondiente a la tercera parte de la población del Departamento. En el año 2009 lo que era el CEAD Neiva hace uso de sus nuevas instalaciones y se convierte en un Centro Comunitario de Atención Virtual CCAV, atendiendo alrededor de 1467 estudiantes.

Se ha destacado por presentar una gran actividad investigativa que le ha permitido a investigadores y estudiantes obtener la financiación en 7 proyectos adscritos a los semilleros Zoociencias, Higea Unadista y Rastreadores de Comunidad según la convocatoria interna realizada por SIUNAD entre los años 2008 y 2009.

De igual modo, a finales del año 2008 el actual CCAV Neiva incuba la propuesta de red de investigación en desarrollo sostenible, que se consolida al año siguiente $\mathrm{y}$, actualmente se caracteriza por tener un gran número de proyectos inscritos a nivel nacional.

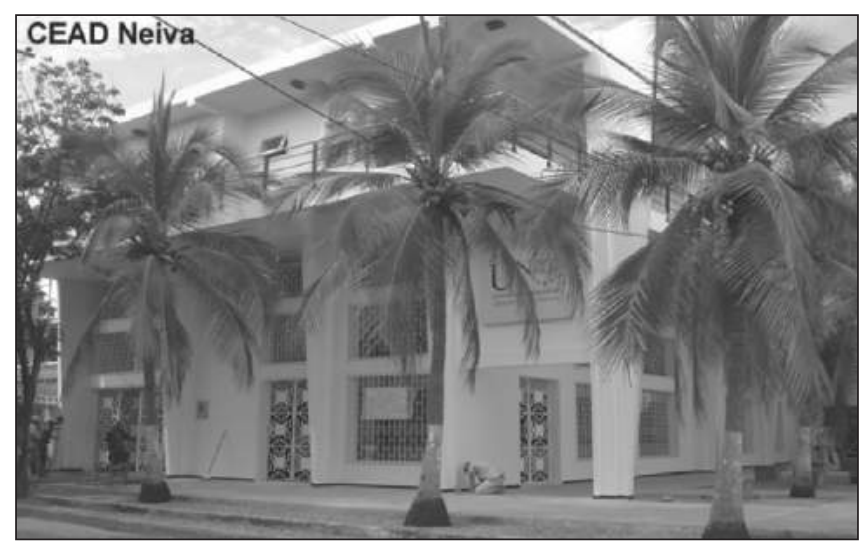

figura 5. CCAV Neiva 


\section{CEAD Pitalito:}

Pitalito está ubicado al sur del Departamento del Huila sobre el valle del Magdalena y en el vértice que forman las cordilleras central y oriental, a $1.318 \mathrm{mts}$ sobre el nivel del mar y a unos $188 \mathrm{Km}$ de la Capital del Huila. Es considerado la Estrella Vial del sur de Colombia, por su localización estratégica, que permite la comunicación con los Departamentos vecinos del Cauca, Caquetá y Putumayo. Posee una extensión total de $666 \mathrm{Km} 2$, su temperatura promedio se encuentra entre $18^{\circ}$ y $21^{\circ} \mathrm{C}$; actualmente el CEAD atiende un número total de 873 estudiantes.

Se ha caracterizado por su liderazgo de proyectos en pro del desarrollo sostenible; en este sentido, el grupo de investigación InYUMACIzo, aunque nació en la Escuela de Ciencias Agrícolas, ha abierto la posibilidad a que estudiantes y tutores de otras escuelas puedan participar de los procesos investigativos.

INYUMACIZO, ha logrado consolidarse como un grupo generador de proyectos de investigación con gran pertinencia para la Zona, lo cual se puede evidenciar en los excelentes resultados en la convocatoria de financiación de proyectos SIUNAD 2009, tales como: Segunda fase de la evaluación comparativa del pasto Maralfalfa, pasto elefante verde y morado en el municipio de Pitalito, Evaluación de cinco parámetros de calidad en fruta de la mora de castilla rubus glaucus benth variedad sin espinas comparada con la variedad con espinas, en cultivos de la zona sur del departamento del Huila y Formulación de un modelo metodológico para la implementación del plan institucional de gestión ambiental para la universidad nacional abierta y a distancia (UNAD), CEAD Pitalito, Huila.

Actualmente el grupo se encuentra formulando dos trabajos de grado pertenecientes al doctorado en desarrollo sostenible: Constitución de la cadena productiva de la guadua para la cuenca hidrográfica del Río Guarapas, Departamento del Huila y Condiciones de calidad y cantidad de recurso hídrico para consumo humano en los principales afluentes del Río Guarapas, Departamento del Huila.

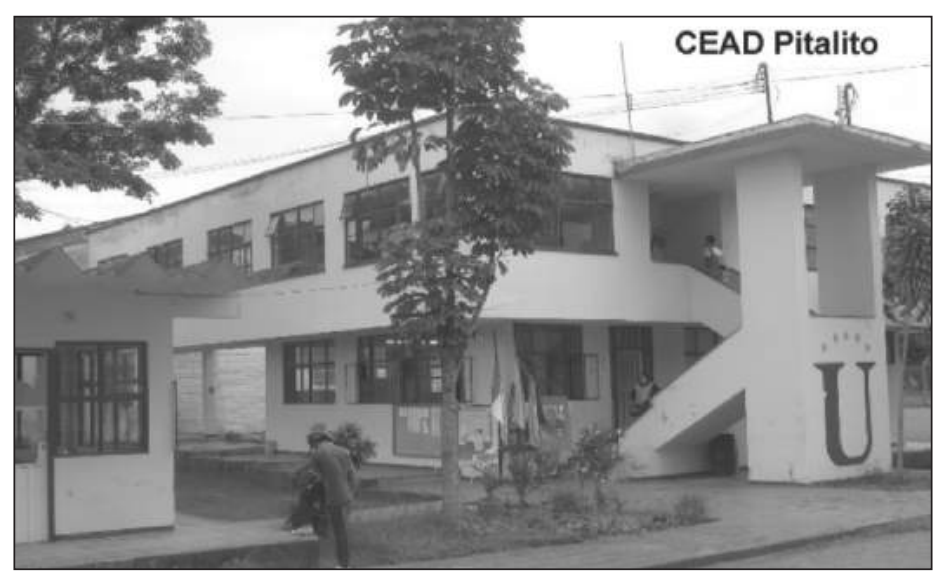

figura 6. CEAD Pitalito 


\section{CERES La Plata:}

La Plata es una población de aproximadamente 55.000 habitantes, incluyendo la zona rural; una altitud de $1.050 \mathrm{msnm}$. Está ubicado a una distancia $122 \mathrm{~km}$ desde Neiva, 147 km de la ciudad de Popayán y a 210 km de la población de San Agustín. El municipio de La Plata se encuentra localizado en la Parte Sur-Occidente del Departamento del Huila, en las estribaciones de la Cordillera Central.

Hoy en día el CERES atiende un número promedio de 334 estudiantes. Sus procesos de investigación han venido creciendo a lo largo de los últimos años, se ha destacado por la participación en las convocatorias internas de SIUNAD a través del grupo Raipla y por el liderazgo presentado en la estructuración del portal web de investigación Zonal w inzo.

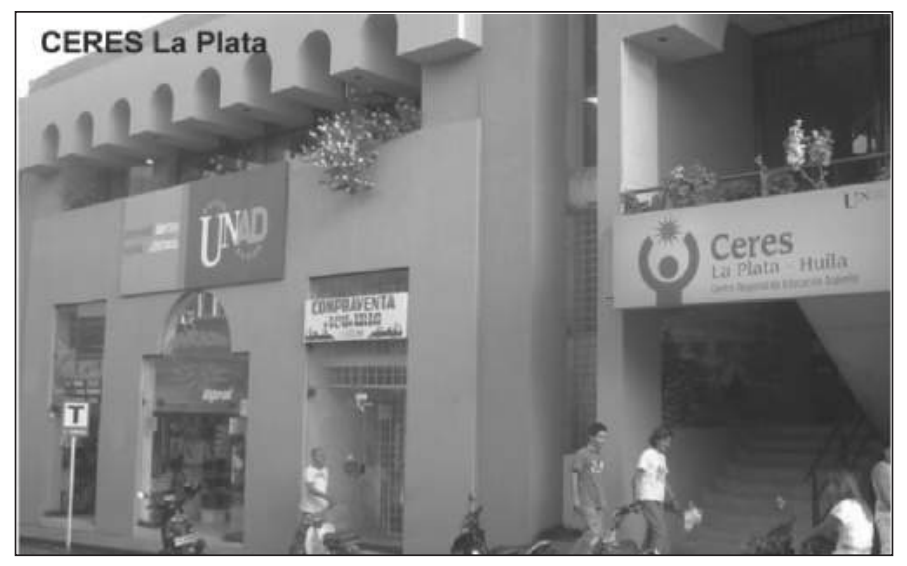

figura 7. CERES La Plata

\section{El Caquetá}

Se encuentra localizado al suroriente del país, siendo Florencia su capital. La región del Caquetá se inicia en el pie de monte andino y termina en los escarpes de Araracuara, en plena selva amazónica. Se destaca por sus hermosos ríos Ajajú o Apaporis, Yarí, Caguán y Orteguaza, navegables por embarcaciones menores.

A excepción de las poblaciones que se hallan situadas en las bases de la cordillera y unos pocos caseríos indígenas en las márgenes de los grandes ríos, el resto del territorio se halla prácticamente deshabitado y cubierto de espesa selva tropical húmeda, con una temperatura de entre $27^{\circ}$ y $29^{\circ}$ C.En este Departamento la UNAD cuenta con un CEAD y un CERES. 


\section{CEAD florencia:}

Florencia, la capital del Departamento de Caquetá, es la ciudad más importante del suroriente colombiano por su número de habitantes, tiene más de cien años de historia y es conocida como "La Puerta de Oro de la Amazonia Colombiana". Está ubicada en el pie de monte entre la Cordillera Oriental y la selva del Amazonas, lo cual le da una posición privilegiada ambientalmente al ser el enlace entre la Región Andina y la Región Amazónica.

Actualmente el CEAD Florencia atiende un número de 994 estudiantes; se destaca por tener dos grupos en categoría D por COLCIENCIAS: Innovación Tecnológica, perteneciente a la Escuela de Ciencias Básicas Tecnologías e Ingenierías (Есвті) у Cananguchales, de la Escuela de Ciencias Administrativas Contables y de Negocios (ECACEN). Estos grupos se han destacado por su gran actividad científica frente a las problemáticas de la región.

El Centro de Atención Psicosocial (CAP) es un grupo de investigación creado desde el año 2008, el cual, se ha convertido en una herramienta fundamental para el desarrollo social de la región. De acuerdo con esto, la UNAD ha podido dar respuesta a las problemáticas de las comunidades más vulnerables de la Zona Sur y ha permitido generar una verdadera inclusión social mediante la investigación y la intervención psicosocial.

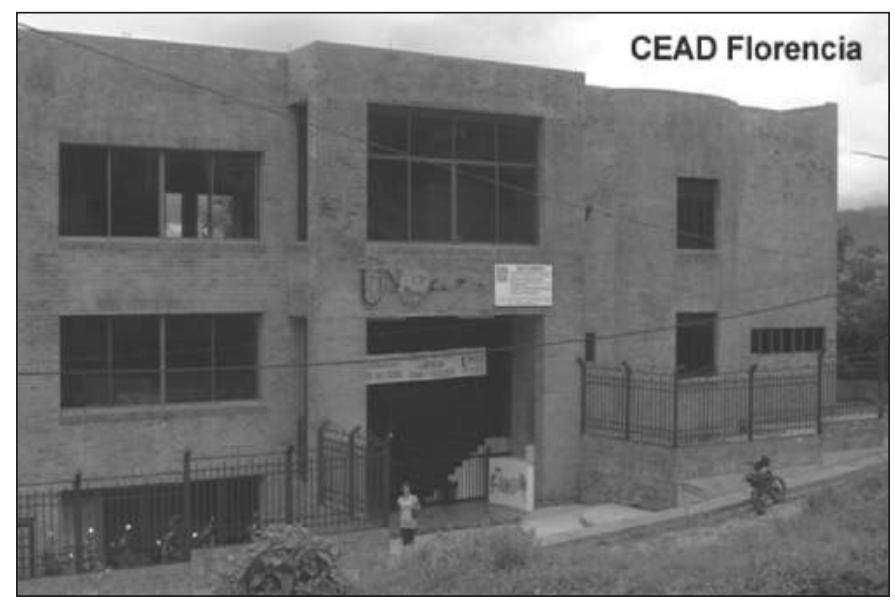

figura 8. CEAD Florencia

\section{CERES San Vicente del Caguán}

Municipio con una población de 3.980 habitantes, se encuentra ubicado a 160 km de Florencia y posee una temperatura de $26^{\circ} \mathrm{C}$. El CERES San Vicente, registra hoy día un número de 192 estudiantes, especialmente pertenecientes a la escuela de Ciencias Agrícolas Pecuarias y del Medio Ambiente (ECAPMA). 
Aunque los procesos de investigación son incipientes, sus tutores y estudiantes han manifestado un gran interés por involucrarse en las actividades de investigación a través de la estructuración de un semillero de investigación perteneciente a ECAPMA.

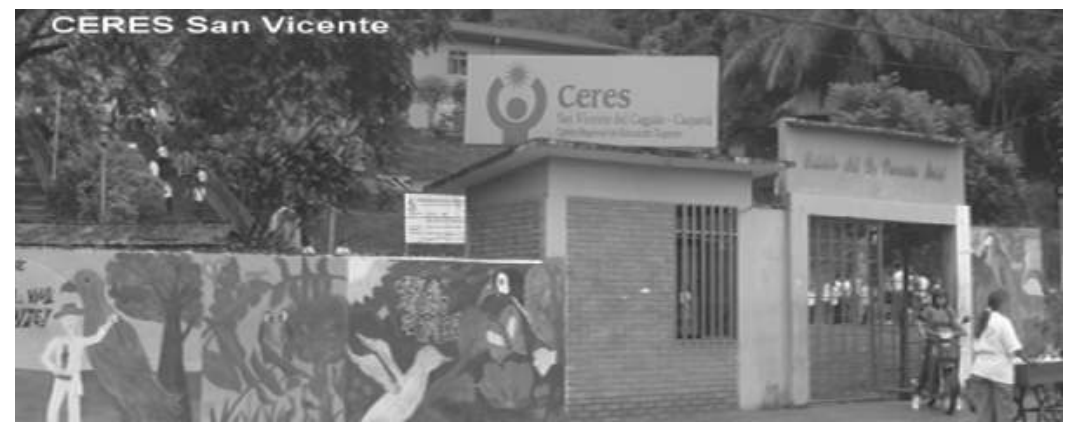

figura 9. CERES San Vicente

\section{Estrategias de consolidación y dinamización del sistema de investigación unadista SIUNAD zona sur}

El reajuste de las zonas y la consolidación de la nueva Zona Sur en el año 2008, permitió encontrar nuevas potencialidades y fuerzas frente a la generación de respuestas a las problemáticas sociales propias de la región. En este sentido y con el fin de poder hacer visible y más participativos los avances investigativos en cada uno de los centros de la zona, se ha identificado un líder de investigación local que permite transferir eficientemente los lineamientos establecidos desde el SIUNAD nacional hacia la comunidad académica en cada uno de los centros.

El gráfico uno, evidencia cómo esta acción ha permitido motivar a la comunidad académica de la zona sur a través del incremento en más de un 50\% del número de tutores vinculados a procesos de investigación con respecto al año 2008.

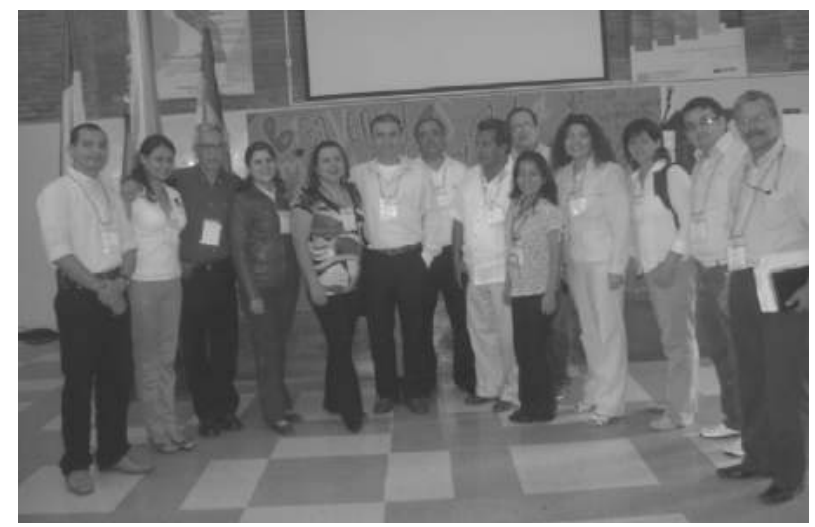

figura 10. Investigadores Zona Sur. I Encuentro Zonal de Investigación Zona Sur. 2009 
Gráfico 1. Comparativo Tutores Investigadores 2008-2009

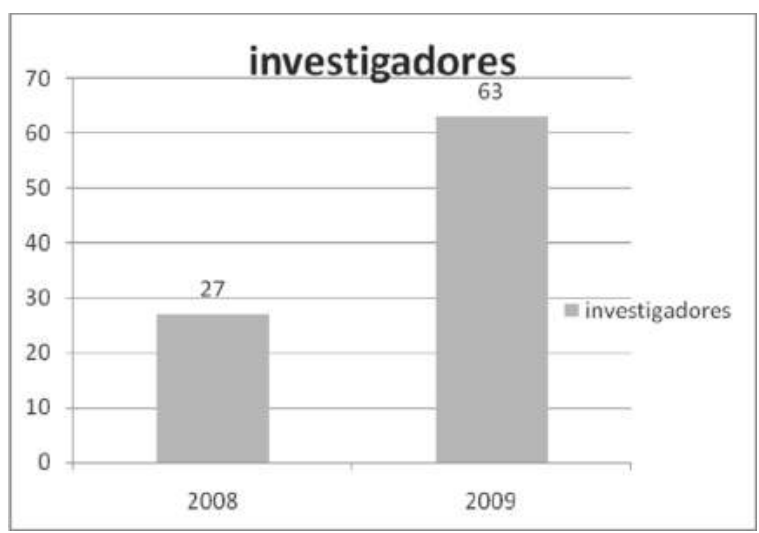

Asimismo, se han logrado estructurar siete nuevos grupos de investigación en todos los CEAD de la zona como lo muestra la gráfica número dos. Este hecho permite revelar la motivación que presenta la comunidad frente a los procesos. Como estrategia para la dinamización del Sistema de investigación Unadista SIUNAD allí, se ha institucionalizado una jornada de investigación a la semana donde se realizan actividad que permite avanzar y hacer control de las metas propuestas por cada uno de los grupos de investigación.

Gráfico 2. Panorama actual de grupos de investigación

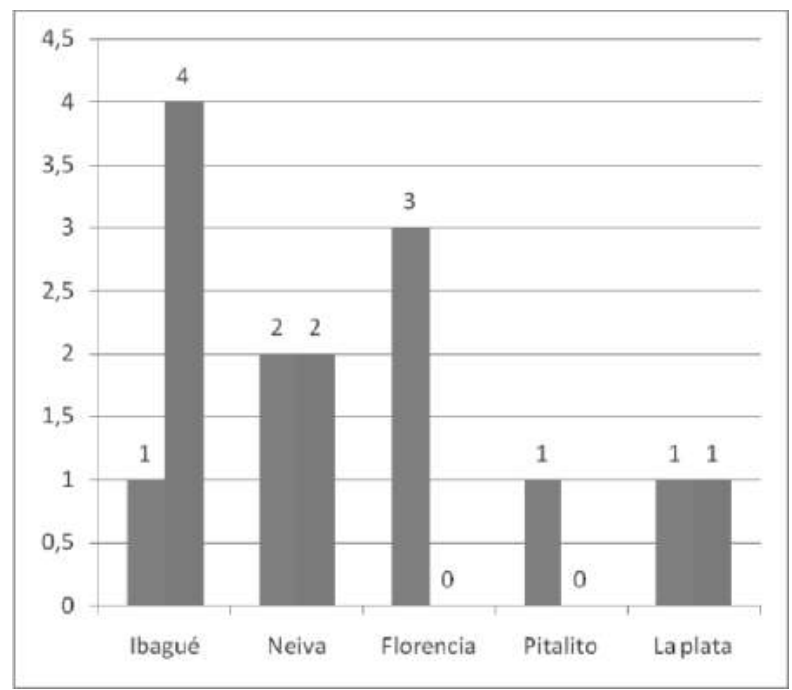


Gráfica 3. Panorama actual de semilleros de investigación

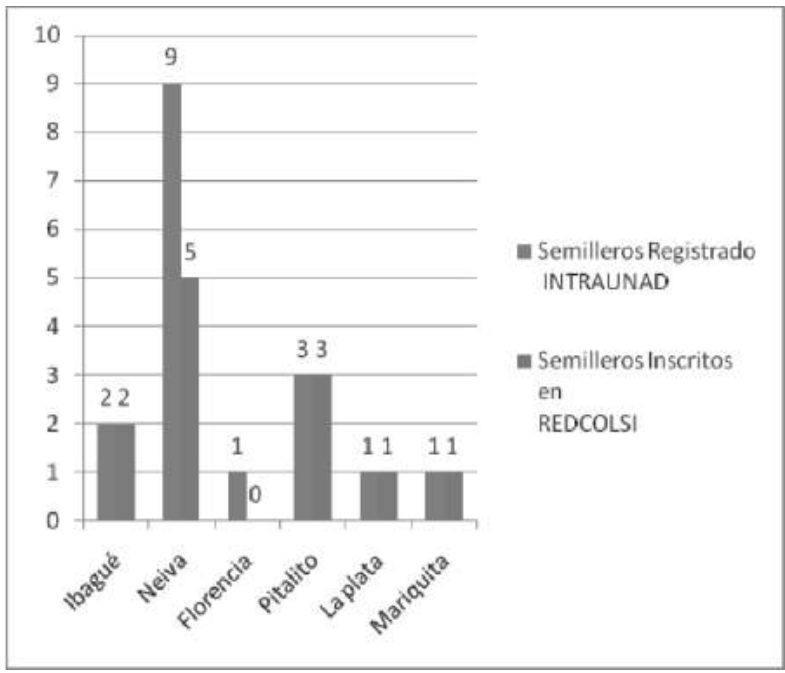

La Zona Sur ha venido generando una verdadera cultura investigativa dentro de sus estudiantes al impulsar la investigación formativa. En este sentido, ha facilitado la conformación de semilleros de investigación, quienes se originan a partir de capacitaciones y socializaciones de resultados en eventos departamentales, zonales y nacionales. (Ver gráfica 3)

\section{La red de investigación en desarrollo sostenible}

En la actualidad, el ser humano en su afán por satisfacer sus necesidades básicas ha hecho uso de los recursos que encuentra a su paso de una forma indiscriminada, olvidando la responsabilidad que tiene con sus generaciones futuras. De acuerdo con lo anterior, se ha planteado la relevancia de satisfacer las necesidades de las generaciones presentes sin comprometer las posibilidades del futuro. Así, el tema de desarrollo sostenible se debe abordar de forma integral con base en tres componentes fundamentales: ambiental, económico y social; el equilibrio entre estos permite llegar a un verdadero desarrollo.

La UNAD, desde la Zona Sur, ha venido formulando de manera significativa proyectos de gran impacto social desde cada una de las Escuelas, gracias al equipo líder de siUnAD, que se encuentra liderando actualmente la red de investigación en desarrollo sostenible.

Los grupos de investigación que inicialmente se han integrado a la red desde la zona son: Buscando Producción, desde el CCAV Neiva; GIEPronal, desde el CEAD Ibagué; Inyumacizo, desde el CEAD de Pitalito, y desde el CEAD de Florencia Cananguchales y El Centro de Atención Psicosocial CAP. 


\section{Prospectiva del desarrollo investigativo en la zona sur}

La Zona Sur se proyecta como una región con respuestas pertinentes a las problemáticas del país, principalmente en los campos de desarrollo sostenible, seguridad y soberanía alimentaria y nuevas tecnologías educativas. En este sentido, los investigadores de la zona se han comprometido con la idea de los Centros de Gestión de Conocimiento Especializado CGCE.

La iniciativa de centros de gestión de conocimiento especializado, es concebida en el año 2009 por la Coordinación Nacional sIUNAD con el apoyo de las Coordinaciones de las zonas Occidente y Sur, como "un dispositivo orientado a que las comunidades se integren y avancen en la sociedad de conocimiento. Es una forma de empoderamiento basado en la gestión de conocimiento vinculada a temas centrales de su actividad productiva y una estrategia orientada a generar desarrollo con equidad social". ${ }^{66}$

En este sentido, la Zona Sur actualmente viene consolidando cuatro propuestas de CGCE.

\section{Apicultura: ${ }^{67}$}

La apicultura es la ciencia que se encarga del manejo de las abejas de la especie Appis mellifera. Su gran importancia radica en la capacidad que tiene el insecto para polinizar los cultivos comerciales.

El departamento del Tolima es una gran despensa agrícola. En los últimos años ha encaminado esfuerzos para desarrollar la fruticultura, la oferta de suelos, clima y vías de comunicación, lo que hace que dicha región tenga ventajas competitivas y comparativas para el desarrollo de este tipo de cultivos. Las zonas sembradas en la actualidad y las nuevas áreas adecuadas con riego en el proyecto "triángulo del sur del Tolima" van a constituir el polo de desarrollo frutícola del País.

Para lograr competitividad en la producción se requiere la utilización de las abejas como agentes polinizadores las cuales, de forma natural, incrementan la producción desde el $20 \%$ en el caso de los cítricos hasta más del $100 \%$ en el caso de cucurbitáceas como melón, sandía y ahuyama. Así, los cultivos son más rentables, incrementan el área demandando mano de obra y esto, a su vez, mejora el desarrollo económico de la región. El liderazgo de este proyecto se encuentra en manos del Grupo de Investigación GIEPRONAL.

66 Maldonado, L. F, Correa, N, Alcócer M. Centro de Gestión de Conocimiento Especializados.2009

67 Ing. Ricardo Tello. Grupo de Investigación GIEPRONAL.2009 


\section{Arracacha: ${ }^{68}$}

Según datos revelados por el ministerio de agricultura, el Tolima es el principal productor de arracacha con el $65 \%$ de la producción nacional. Cabe resaltar que de este porcentaje departamental, el 90\% corresponde al municipio de Cajamarca.

Cajamarca es un municipio ubicado sobre la Cordillera Central a 45 minutos de la ciudad de Ibagué y $420 \mathrm{Km}$. de Bogotá. Cuenta con 42 veredas, es netamente agrícola debido a su riqueza hídrica y suelos propicios para tal fin, cuenta con una temperatura promedio $20^{\circ} \mathrm{C}$, con una altura de 1700-2300 msnm, su economía se centra en cultivos de gran importancia como la arracacha (Arracacia xanthorriza) hortaliza que se produce en los valles interandinos y otras regiones del país, importante en la alimentación por la fácil digestión de sus almidones y por ser rica en calcio, fósforo, hierro, niacina, vitamina A, piridoxina-B6, riboflavina-B2, ácido ascórbico, proteínas, fibras y carbohidratos; características que le otorgan un potencial alimentario y económico. La parte aérea está compuesta por un conjunto de brotes y hojas que son utilizados en la preparación de nuevas plántulas destinadas a la propagación vegetativa o en la alimentación de bovinos y equinos.

Tecnológicamente se están adelantando desarrollos de innovación a partir de la obtención en harina y su transformación en Arequipe y Torta de Arracacha, por parte de un grupo de mujeres ubicadas en el corregimiento de Anaime con apoyo Científico del grupo GIEPRONAL y del semillero BIOTECAL.

\section{Piscicultura: ${ }^{69}$}

La acuicultura, según la FAO, se presenta como la fuente alternativa de proteína para la seguridad alimentaria mundial. En los últimos 20 años la producción mundial piscícola ha aumentado significativamente; la tilapia,la trucha y la cachama se destacan por su crecimiento a ritmos de 12\%, 6\% y 29\% respectivamente (Acuerdo de Competitividad Cadena Piscícola, 2005).

En lo referente al departamento del Huila, se ha presentado un comportamiento creciente en la superficie dedicada a la actividad. Se destaca el municipio de Aipe con un crecimiento anual de 67\%, seguido por el Embalse (Campoalegre, Yaguará y Hobo) con un crecimiento cercano al $60 \%$. Todo lo cual ha redundado en un aumento anual de $25 \%$ en el área en espejo de agua en el departamento.

Las especies que se encuentran difundidas entre piscicultores (especialmente los pequeños y que producen para autoconsumo), están: la carpa, la mojarra plateada y la cachama, cuya producción se hace con un mínimo o casi nulo suplemento alimenticio de concentrados en algunos casos. No obstante, con el apoyo de la Secretaría de Agricultura, complementan con

68 Esp. Milena Alcócer Tocora. Grupo de Investigación GIEPRONAL, Semillero BIOTECAL. 2009

69 Ing. Deya Amparo Perez Zuñiga. Grupo de Investigación Buscando Producción, Semillero Bio-logicos.2009 
fuentes alternativas como son las larvas de moscas, lombriz de tierra, la azola, el bore y el ramio, entre otras.

El montaje del centro ha sido liderado desde el año 2009 por el grupo de investigación Buscando Producción y el semillero Bio-lógicos.

\section{Seguridad Alimentaria: ${ }^{70}$}

Colombia, un país con 1'141.748 kilómetros cuadrados donde habitan 42.3 millones de personas, espera para el 2020 una población de 57 millones y 60 millones para el 2025. En este sentido se ha generado la siguiente pregunta: ¿Cómo mejorar las condiciones generales de la población, entre ellas la seguridad alimentaria, teniendo en cuenta aspectos como la distribución de la propiedad y del ingreso, la situación política, y social por la que el país atraviesa, la institucionalidad, la sostenibilidad, y, en general, el equilibrio de la sociedad en su conjunto?

La posibilidad real de la población para acceder a una oferta permanente de alimentos derivados tanto de la producción agropecuaria interna como de lo que pueda conseguirse en el mercado mundial para satisfacer sus necesidades, que garantice la estabilidad en la provisión alimentaria en términos de cantidad y calidad debe ser una responsabilidad de la sociedad científica y de la comunidad en general.

Tendencias mundiales para la distribución de consumo:

- Para el año 2025 se espera que alrededor de cinco mil millones de personas se encuentren viviendo en las ciudades, representando el 59\% de la población mundial. Para el año 2050 se esperan 9.200 millones de personas en el pequeño planeta tierra.

- “Un nivel decente de vida, nutrición suficiente, atención de salud, educación, trabajo decente y protección contra las calamidades no son simplemente metas de desarrollo, son también derechos humanos". PNUD 2000.

- En Colombia el gasto en programas de nutrición es de 1,63\% del gasto público.

Actualmente este proyecto de CGCE se encuentra liderado por el grupo INYUMACIZO en el Huila.

De otro lado, la zona pretende avanzar en la visibilidad de su producción científica aumentando la cantidad de productos científicos publicados; para el cumplimiento de este objetivo, la zona con el apoyo de la Escuela de Ciencias de la Educación ECE, se encuentra diseñando un curso de producción de artículos científicos.

70 Esp. William Montealegre. Grupo de Investigación INYUMACIZo.2009 
Para el año 2010 la Zona será sede del "V Encuentro Nacional de Investigación” aportando significativamente a la integración y consolidación del Sistema de Investigación Unadista SIUNAD desde las regiones.

\section{AGRADECIMIENTOS}

La coordinación zonal de investigación hace un reconocimiento especial a la dirección zonal y a cada uno de los directores de centro por todo el apoyo recibido para la consolidación de los procesos y destaca en gran medida el compromiso y el trabajo realizado por cada uno de los investigadores y estudiantes de la zona. De esta forma, se ha entregan al proyecto UNAD invaluables aportes que permiten cumplir con su misión y visión institucional.

\section{REfERENCIAS BIBLIOGRáfICAS}

MALDONADO, L. F., CORREA N, ALCóCER M. (2009). Centros de Gestión de Conocimiento Especializados. UNAD.

PERIóDICO UNIENDO DISTANCIAS. (2010). Zona Sur. Investigación y Región. Abril,

DESCRIPCIóN DE DEPARTAMENTOS DE COLOMBIA. Disponible en: http://www. todacolombia.com/departamentos. 